\title{
Investigación cuantitativa en una práctica universitaria de intervención en comunidad en el norte de México
}

\author{
Adan Cano-Aguilar \\ Licenciado en Sociología. Doctor en Filosofía \\ Universidad Autónoma de Ciudad Juárez. Juárez, México \\ https:// orcid.org/0000-0001-8809-2189 • adan.cano@uacj.mx
}

Resumen

En este artículo se presentan los resultados de una encuesta aplicada en un barrio de Casas Grandes, Chihuahua, en el norte de México, en el marco de un trabajo de diagnóstico social e intervención comunitaria realizado durante 2017. Se comparte la experiencia de la aplicación de este instrumento de recolección de datos cuantitativos y el análisis de los mismos, a fin de identificar áreas de intervención, en el contexto del desarrollo social y económico de la región que tiene como epicentro la zona arqueológica de Paquimé, adyacente a la comunidad de referencia y la más importante en el norte del país. Se presentan los resultados del análisis de los datos obtenidos en tres apartados: características y condiciones de bienestar de los colonos, disponibilidad e interés para proyectos de promoción social y representaciones del contexto de desarrollo. Se expone la metodología y el análisis descriptivo estadístico, en el contexto del Trabajo Social comunitario. Se resaltan las posibles aportaciones de los resultados de esta encuesta al diagnóstico integral de la comunidad, el mismo que contempla otras técnicas cualitativas y cuantitativas de recolección de información.

Palabras clave: Comunidad; Trabajo Social; Práctica universitaria; Diagnóstico social; Investigación cuantitativa.

Recibido: 26/06/2019 | Aprobado: 20/11/2019

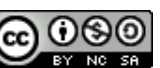

Esta obra está bajo una Licencia Creative Commons Atribución-NoComercial-CompartirIgual 4.0 Internacional.

Financiación o proveniencia del artículo: Artículo derivado del proyecto Diagnóstico social y sensibilización realizado en la Colonia Progreso en Casas Grandes, Chihuahua, desarrollado en el periodo octubre de 2016 - diciembre de 2017, financiado por el Gobierno municipal de Casas Grandes y Universidad Autónoma de Ciudad Juárez, México.

¿Cómo citar este artículo? / How to quote this article?

Cano-Aguilar, A. (2020). Investigación cuantitativa en una práctica universitaria de intervención en comunidad en el norte de México. Prospectiva. Revista de Trabajo Social e intervención social, (29), 107130. doi: $10.25100 /$ prts.v0i29.8196. 


\title{
Quantitative research in a scholar practice of community intervention in northern Mexico
}

\begin{abstract}
This article presents the results of a survey applied in a neighborhood of Casas Grandes, Chihuahua, in the north of Mexico, in the framework of a work of social diagnosis and community intervention carried out during 2017. The purpose is to present the experience of the application of this quantitative data collection instrument and its analysis, in order to identify areas of intervention, in the context of the social and economic development of the region that has as its epicenter the archaeological zone of Paquimé, near to the community and the most important in the north of the country. The results of the analysis of the data obtained in three sections are presented: characteristics and welfare conditions of the settlers, availability and interest for social promotion projects and representations of the development context. The work aims to illustrate the methodology and the descriptive statistical analysis, in the context of community social work. The possible contributions of the results of this survey to the integral diagnosis of the community are highlighted, which contemplates other qualitative and quantitative data collection techniques.
\end{abstract}

Keywords: Community; Social work; Scholar practice; Social diagnosis; Quantitative research.

Sumario: 1. Introducción, 2. Metodología, 3. Hallazgos, 3.1. Características socio demográficas y de bienestar social, 3.2. Participación y organización comunitaria, 3.3. Representaciones sociales del contexto inmediato, 3.4 Potenciales áreas de intervención identificadas, 4 . Conclusiones, 5. Referencias bibliográficas. 


\section{Introducción}

En la práctica del Trabajo Social el concepto de diagnóstico ha recibido múltiples denominaciones: valoración, evaluación, dictamen técnico, juicio diagnóstico, juicio profesional, interpretación diagnóstica, valoración técnico-científica, opinión profesional, etc. (Cury y Arias, 2016). Así, en el período en que el Trabajo Social profesional estaba centrado en el estudio de casos y problemas de individuos y pequeños grupos desde un enfoque psicosocial y clínico, el término "diagnóstico" tomó sus referencias iniciales: se deberá identificar y priorizar el problema antes de prescribir el tratamiento. Más allá de sus encuadres teórico metodológicos, en términos prácticos el diagnóstico implica contar con información precisa y pertinente acerca de un problema a atender. Se ha señalado esta función o finalidad del diagnóstico para "obtener conocimiento certero para lograr acciones que conduzcan al cambio en función de las necesidades e intereses de los actores sociales" (Arteaga y González, 2001, p. 89).

En los niveles de la intervención de caso y de grupo, se deben considerar sus necesidades e intereses como profesionales de la intervención social, los de las personas que reciben la acción (individuos o grupos pequeños) y los de las instituciones involucradas usualmente en estos niveles de intervención (Viscarret, 2009). En un ámbito de intervención más amplio, el diagnóstico debe arrojar información que permita proyectar y orientar acciones para la atención de problemas; los ejes de la investigación diagnóstica son los problemas en la comunidad y sus factores; las necesidades, debilidades y amenazas de los pobladores; los recursos, medios y oportunidades con las que cuentan (Lillo y Roselló, 2004). En el ámbito del Trabajo Social comunitario existen dos niveles característicos de estudio e intervención social: la organización comunitaria y el desarrollo comunitario. El primero requiere de mecanismos de participación y de un nivel de cohesión social; el segundo implica sobrepasar el campo de la acción social, se refleja en la calidad de vida y el bienestar de los integrantes de la comunidad y se atienden necesidades de la comunidad como un sujeto social (Eito y Gómez, 2013).

Carballeda (2013) resalta el componente de la integración comunitaria implícito en el primer nivel de intervención: "la cuestión de la identidad, relacionándose con una necesaria mirada a los lazos sociales" (p. 11); de manera que se articulan organización, participación y desarrollo comunitario. Barbero y Cortés (2005) opinan que de los tres ejes de trabajo comunitario (concientización, organización y movilización), el de organización comunitaria es el más apropiado para comprender este ámbito de intervención del Trabajo Social. En el concepto de comunidad, se han identificado dos dimensiones con sus lógicas correspondientes: la teórica y la operativa. En esta última se circunscriben los elementos y aspectos de la práctica cotidiana de intervención:

Siguiendo el análisis histórico de Hillery (1955) éstos serían tres: (i) espacio o territorio compartido, (ii) relaciones y (iii) pautas de interacción. Revisando otras definiciones como las de Marchioni (2004) o Nogueiras (1996) podríamos ampliar estos elementos clave con los (iv) recursos que posee la comunidad y las (v) demandas que surgen en su propio seno. (Eito y Gómez, 2013, p. 13). 
Además, entre las tareas regulares y habituales que los trabajadores sociales comunitarios deben desarrollar está la de "Descubrir necesidades y potencialidades del espacio social de que se trate (barrio, institución, un colectivo social, etc.)" (Barbero y Cortés, 2005, p. 20). De modo que el diagnóstico ha sido definido, en algunos manuales de intervención comunitaria, como

el punto de partida para la formulación de un proyecto (...) identificar cuáles son los problemas, las necesidades que aquejan al territorio y a las familias que lo pueblan, como así también lo que tenemos. Aquí es bueno preguntarnos cómo vivimos, qué hacemos, qué tenemos, qué producimos, qué nos falta. (Instituto de Investigación y Desarrollo Tecnológico para la Agricultura Familiar - IPAF NOA INTA, 2010, p. 13).

El diagnóstico social es útil para capturar "el punto de vista de los miembros de la comunidad, con la colaboración de los diferentes colectivos y partes interesadas. Produce una representación colectiva del espacio comunitario, que sirve de catalizador para poner en marcha acciones concertadas" (Maya, 2016, p. 4). También es apropiado destacar los criterios para el diagnóstico social comunitario aportados por Lillo y Roselló (2004): contar con una visión global de la realidad; abiertos a la inter multidimensionalidad; procurando la coherencia de la intervención con los fines que se planteen; promoviendo la participación de los agentes intervinientes y la visibilidad. Estos principios apuntalan diversos fundamentos metodológicos y procedimentales, entre ellos: una perspectiva integral de los diversos aspectos de la comunidad, el vínculo entre instrumentos y objetivos del proyecto y la identificación y acompañamiento de actores externos de la comunidad (autoridades, organizaciones, academia, etc.).

Se han delimitado y definido momentos sustancialmente distintos del proceso de diagnóstico. En un primer paso, de investigación propiamente, se estudian los problemas y sus factores, y el contexto de la problemática. En una segunda fase se analizan los recursos disponibles y los factores más influyentes. En un tercer momento se proyectan las tendencias del problema, se deciden prioridades y estrategias y se reconocen los factores de viabilidad y factibilidad de la intervención (Aguilar y Ander-Egg, 1999, p. 20).

En los pasos capitales del método expuesto por Mori (2008) se despliegan diversas técnicas de recolección y tratamiento de información. En un primer paso, se revisa la información disponible acerca de la localidad que se quiere intervenir; después, se realiza el mapeo y lotización, el barrido de la información:

recorrer la comunidad y registrar lo que a modo de infraestructura encontramos, es decir registrar el número de viviendas (lotes, manzanas), espacios de recreación, locales de las organizaciones de base y describir el estado en el que se encuentran; así mismo a medida que avanzamos en el recorrido, vamos construyendo un mapa de recursos, con los que podremos contar en el proceso (...) a entablar primeros contactos con los actores de la comunidad, en tanto los miembros de la comunidad empiezan a conocer a los interventores. (Mori, 2008, p. 84).

También se realiza una tercera etapa de construcción de instrumentos de recolección de datos y luego viene el análisis de la información. Por último, se hace énfasis en el hecho de que el concepto de comunidad en la dimensión práctica y operativa puede aludir a tres 
niveles de sentido: lo comunitario como encuadre demográfico territorial; como conjunto de relaciones y espacio de exclusión - inclusión social; y como motor de cambio (Eito y Gómez, 2013). En este trabajo, el contexto de la intervención obligó a ceñirse a la primera de estas tres categorías.

En el municipio de Casas Grandes, en el estado de Chihuahua, ubicado en la frontera noroeste de México con El Paso, Texas, Estados Unidos, se encuentran recursos naturales, históricos, arquitectónicos y culturales que conforman un potencial importante para el desarrollo económico y social de esta región noroeste del estado, conformada además por los municipios de Janos, Nuevo Casas Grandes, Galeana, Buenaventura y Ascensión; situados en una zona geográfica de transición del sistema serrano al altiplano semiárido. La problemática de la violencia se ha manifestado de manera dramática en esta región noroeste, coincidentemente con la ejecución de las políticas de seguridad nacional en 2007 (Cano, Estrada y Chávez, 2017). Sin embargo, a partir de 2012, disminuyó la intensidad de la violencia, la cual ha sido contenida, y la dinámica socio económica de la región parece haberse adaptado a las nuevas condiciones, conformándose un nuevo nivel de normalización de la problemática.

La reciente inscripción del municipio de Casas Grandes en el Programa federal de "Pueblos Mágicos" abrió una oportunidad y coyuntura para incidir en la atención de necesidades de desarrollo económico y social de las comunidades de esta región, desde un enfoque de turismo cultural. En este Programa, de desarrollo turístico "para localidades que en un diferente nivel de desarrollo, [se] organizan diversas acciones de carácter económico, social y ambiental con el propósito de mejorar las condiciones de vida de una localidad turística" (SECTUR, 2017, p. 24), para lo cual se busca promover la participación de los habitantes en la preservación de los patrimonios natural, cultural e histórico de estas localidades, a través de la consolidación y creación de oferta turística atractiva; aprovechando las particularidades de las localidades para la generación de productos turísticos basados en la cultura local (artesanías, festividades, gastronomía, y tradiciones); promoviendo que las comunidades de las localidades "aprovechen y se beneficien del turismo como actividad redituable como opción de negocio, de trabajo y de forma de vida" (SECTUR, 2006, p. 2).

Mediante entrevistas y conversaciones realizadas desde 2015, se recogieron diferentes puntos de vista de autoridades del gobierno local, miembros del comités ciudadanos y artistas y artesanos de la cabecera municipal de Casas Grandes. Se detectó la necesidad de crear las bases para contribuir a aportar valores agregados a la oferta turística existente, mediante la generación de información, y la sensibilización, la promoción y la participación social de los habitantes. Además, se fomentaría la sostenibilidad sociocultural, al buscar "hacer de la actividad turística algo duradero, y sobre todo, rentable sin descuidar aspectos fundamentales como el ambiente y la cultura" (SECTUR, 2015, p. 21), promoviendo intercambios culturales entre turistas y habitantes.

En este contexto se consideró apropiada una propuesta de intervención social comunitaria ligada al ámbito del turismo sostenible, debido a que la actividad turística, como otras ramas de la economía regional, se vio afectada por la violencia y la inseguridad de fines de la última década, por un lado; y por otro, a que el horizonte que se abrirá en la 
región cuando se concluyan las obras de infraestructura vial que el gobierno federal y estatal realizan para conectar esta región con el estado de Sonora, en las costas del Pacífico, conformará una amplia ventana de oportunidades al sector, las cuales podrían ser aprovechadas por los habitantes de este pueblo, principalmente por aquellos que residen en el centro de la localidad o cerca de la zona arqueológica.

En el contexto académico particular en el que se sustenta este artículo, las prácticas de intervención de Trabajo Social en comunidad se habían realizado siempre bajo un modelo de acción social, donde el o la practicante de Trabajo Social es un agente de las instancias públicas para realizar ciertas acciones en cierta localidad (llamada genéricamente "comunidad", para distinguirla del nivel de grupo y caso), sobre todo de infraestructura; o como un mediador entre estas instituciones y la comunidad, como un gestor externo de recursos para la comunidad, principalmente en el ámbito del equipamiento urbano.

En un trabajo de sistematización de una práctica de comunidad en 2012, se expone: que la intervención se guió bajo el modelo de Investigación Acción Participativa (IAP); que se seleccionó la comunidad a intervenir mediante la técnica de "lluvia de ideas", es decir, las estudiantes propusieron localidades, de acuerdo a lo que creían o sabían de sus problemas más importantes; que se aplicaron diversas técnicas de recolección, "como la observación, recorridos en vehículo y caminando, encuestas aplicadas a las personas de la colonia e investigaciones hechas a instituciones como Presidencia Municipal" (Maldonado, 2013, p. 17). Como resultado de estas actividades, se encontró que

los problemas que se tenían con la electricidad, y que el servicio del agua no llegaba de forma adecuada a todos los hogares, los practicantes informaron que se había investigado en presidencia municipal y se informó que no se podrían poner los servicios ya que la colonia era irregular y se debía pagar titulación y terreno, los habitantes respondieron que ya se había pagado 8 mil pesos por terreno y 1100 pesos por titulación, pero que estaban de acuerdo en llegar a un acuerdo con presidencia, los practicantes propusieron acordar una reunión con personal de presidencia y habitantes para la aclaración de dudas y estuvieron de acuerdo. (Maldonado, 2013, p. 56).

A pesar de este dato, las actividades que se realizaron durante la intervención, no estuvieron dirigidas a atender este problema más relevante del diagnóstico, limitándose a gestionar y realizar en la comunidad actividades de servicios de salud y entretenimiento; y tampoco se ciñeron al modelo de la IAP.

En otro ejemplo, en la sistematización de una práctica de comunidad realizada en 2014, se expone que, después de haber realizado entrevistas estructuradas, "recorridos sensoriales" y encuestas, y contar con un diagnóstico, se procedió a programar

diferentes visitas como en el Departamento de Tránsito aprobando la intervención de las practicantes, sin embargo, no se tenía algún material que beneficiara a la intervención, por lo que se optó por visitar a Presidencia [municipal], con el objetivo de presentar a las practicantes. Se dio como resultado, la aceptación por parte del secretario y el apoyo pertinente que se suscitara, aportando de manera ágil la gestión de las láminas para los señalamientos viales. (Ruiz, 2016, p. 19).

Prospectiva. Revista de Trabajo Social e intervención social • No. $29 \bullet$ ene.-jun. 2020 • pp. 107-130 e-ISSN: 2389-993X • Doi: 10.25100/prts.v0i29.8196 Universidad del Valle - Cali, Colombia 
En la práctica de comunidad que sustenta este artículo, se partió de un modelo de desarrollo comunitario desde adentro, en el que se considera que la intervención social en comunidad debe agotar los niveles de organización, participación y desarrollo comunitario, de manera que no se concibe la intervención como una acción de gestión o en la que los colonos sean meros receptores pasivos de los beneficios traídos por los practicantes de Trabajo Social, sino protagonistas del desarrollo comunitario.

Por lo tanto, los tres apartados analizados en este artículo son pertinentes al ámbito del Trabajo Social en comunidad, y conforman una base de información sobre la cual diseñar, implementar y evaluar acciones específicas y concretas que incidan en alguno de los niveles de intervención de este ámbito, y, más importante aún, identificar a las personas de la comunidad con quienes se pueden llevar a cabo estas acciones. Por otro lado, esta técnica permite también identificar posibles obstáculos o dificultades (como la falta de cohesión entre los vecinos), que tendrían que ser atendidos antes de iniciar cualquier acción de intervención.

\section{Metodología}

En el municipio de Casas Grandes residían 10,857 personas, y en su cabecera municipal residían 5,256, de acuerdo con el último censo oficial de 2010 (INEGI, 2010). En la cabecera municipal, la colonia Progreso se encuentra ubicada al suroriente, en sus límites con el agostadero ejidal y la zona arqueológica de Paquimé, desde 1998 nombrada un Patrimonio de la Humanidad por la UNESCO. Se eligió hacer el diagnóstico en esta colonia, porque no se habían realizado aquí diagnósticos ni acciones de Trabajo Social comunitario y por su potencial de desarrollo por estar ubicada frente a esta zona arqueológica y al museo de sitio, que conforman el sitio arqueológico más visitado en esta región del norte de México (INAH, 2016).

En el centro de esta colonia hay un parque en el que se encuentra un espacio para practicar algunos deportes, una cancha para jugar basquetbol o fútbol rápido, áreas verdes y un kiosco. El parque además cuenta con la iglesia de Nuestro Señor de la Misericordia, una iglesia que semeja un templo colonial católico. En la colonia también se encuentran las residencias y estudios de algunos artistas, una de ellas construyó su estudio de pintura con diferentes tipos de botellas de vidrio, el cual es visitado por turistas locales y foráneos y conocido como la "casa de las botellas". La colonia está delimitada al norte por la carretera que conduce a los sitios de interés turístico del municipio: ex haciendas, un pueblo productor de ollas de barro pintadas, una antigua colonia mormona y algunos parajes arqueológicos y naturales.

El objetivo general del proyecto fue realizar un estudio diagnóstico en la cabecera del municipio que generara información para diseñar estrategias que atendieran áreas específicas identificadas como coadyuvantes del desarrollo económico y social de la localidad. Se trataba de contribuir a echar a andar mecanismos de desarrollo desde adentro, aprovechando las capacidades y recursos de la población, incorporándola a los planes de desarrollo, creando y organizando pequeños grupos con potencial para crear nueva oferta turística en el mediano plazo, que promueva nuevos procesos culturales, económicos y sociales, en el marco del desarrollo sustentable. Los objetivos específicos de este proyecto 
son coherentes con la definición de turismo sustentable (Colorado, 2015): hacer un diagnóstico social en la zona aledaña al centro del pueblo y a la zona arqueológica en la cabecera municipal, con la finalidad de 1) conocer las características de las unidades domésticas, necesidades y percepciones de los habitantes y sus recursos productivos de los habitantes y proponer acciones para su aprovechamiento en actividades productivas sostenibles; y 2) identificar, revalorar y reconocer tradiciones, prácticas, costumbres, oficios y expresiones artísticas y culturales de la localidad.

Para alcanzar estos objetivos, se requirió recabar información a partir de datos cuantitativos y cualitativos, por lo que se diseñaron y aplicaron diversas técnicas de recolección. Se elaboró una monografía del municipio, resaltándose sus aspectos económicos, naturales, históricos y culturales; se reunió, ordenó y presentó información documental confiable y actualizada sobre las principales características del contexto del diagnóstico.

También se usó una técnica grupal de recolección de información, que permitió consultar con empresarios, autoridades y personas clave de la localidad los temas del diagnóstico. Las sesiones se realizaron en la universidad, en público, en el formato de paneles, donde hay un moderador que hace preguntas a los participantes y al final se abre un espacio para preguntas del público. Se hicieron dos paneles, uno con autoridades del gobierno local (directores de Turismo, Desarrollo rural, Obras públicas, Cultura, Desarrollo social) y otro con empresarios y promotores culturales y turísticos de la región. El propósito de la técnica fue obtener información de expertos o personas clave de la comunidad con respecto a sus percepciones de las necesidades y problemas y recursos de la comunidad.

Se realizaron también recorridos individuales o en grupos por la localidad, cuyo propósito fue reconocer el perímetro y calles principales, las condiciones del equipamiento urbano, características de espacios públicos y domicilios; construir mapeo de la localidad; identificar diferencias dentro de la localidad (parque; zona arqueológica, carretera, etc.). Las observaciones se registraron en un diario de campo. Estos recorridos también servirían para familiarizarse con las zonas de aplicación del instrumento cuantitativo.

Posteriormente, se empleó una técnica cuantitativa: se aplicó una encuesta en hogares. En este componente del trabajo de diagnóstico se refleja un modelo de diagnóstico de tipo tradicional y no participativo, si se le considera de manera aislada; sin embargo, hay que considerar las aportaciones y relevancia de esta técnica, tomando en cuenta que se encuentra ubicada en un continuum de técnicas desplegadas, antes y después. Como ya se ha mencionado, antes de aplicar esta técnica, se han utilizado otras previamente, como recorridos exploratorios por la zona de estudio, entrevistas con personajes clave del municipio (empresarios, autoridades, promotores representantes de organizaciones locales) acerca de esta colonia, análisis de documentos (mapas, noticias, publicaciones en línea, etc.), elaboración de monografías, entre otras. Por otro lado, la información provista por esta técnica será la base para el diseño y aplicación de subsecuentes técnicas de recolección de información, que permitirá terminar un diagnóstico integral y efectivo para la intervención social participativa en la comunidad. Esta técnica, que se ha empleado en el diagnóstico social, consiste en 
un cuestionario diseñado en un orden determinado, que contiene preguntas con un número limitado de respuestas eventuales por parte del entrevistado, como "Sí, "No" o respuestas muy breves. Las preguntas deben responder a variables que se pretenden medir u observar, previamente definidas. (Crespo, 2011, p. 14).

Se diseñó un instrumento para recoger datos cuantitativos de la población del asentamiento seleccionado, el cual incluía preguntas con respuestas cerradas, preguntas abiertas y preguntas de escala. Los objetos de estudio del instrumento fueron las condiciones, percepciones, actitudes, experiencias y expectativas. Se contaría así con una base de datos que puede ayudar a mejorar las acciones de instituciones en este sector de la cabecera municipal, y sustentar el financiamiento de políticas públicas para esta comunidad.

En el diseño del instrumento se consideraron 8 apartados que reconocen las recomendaciones de Mori (2008) con respecto al contenido y definición de variables:

Datos sociodemográficos: Edad y sexo, composición del hogar y de la familia; estado civil, ocupación, desempleo, (...) tipos de vivienda, condiciones de hacinamiento, (...) Características socioculturales: organización de la comunidad, (...) actitudes y conductas sociales, costumbres. Educación: nivel educacional, (...) población estudiantil. Salud: enfermedades frecuentes, centros de salud, acceso al centro de salud. Recursos: organizaciones internas y externas de base, (...) instituciones, grupo de apoyo... Problemas (...). Necesidades: Son las carencias que tiene la comunidad, suele estar asociado a los problemas. (Mori, 2008, p. 84).

Los objetivos de la encuesta fueron: describir las características socio demográficas de los habitantes de la localidad delimitada (colonia Progreso); describir las necesidades y demandas de estos habitantes, en relación a aspectos diversos del bienestar social; identificar sus percepciones con respecto a la localidad (región, municipio, colonia), su bienestar social; y sus expectativas económicas y de bienestar social; estimar el nivel de participación de los habitantes en asuntos de la comunidad o localidad, sus percepciones sobre este tema y su actitud y disposición a participar; identificar los recursos y activos de los habitantes, susceptibles de ser aprovechados en actividades microeconómicas de tipo colectivo, cooperativo o familiar, que sean acordes al potencial económico de la localidad.

El uso de la encuesta puede ser una estrategia útil en el Trabajo Social comunitario; aún en modelos donde se privilegia la recolección de datos cualitativos a través de recorridos, entrevistas, asambleas y otras técnicas grupales (Millán y Pinedo, 2014; Mori, 2008). Además de recoger información socio demográfica y del bienestar social de los habitantes, las preguntas concernientes a la intervención comunitaria que guiaron esta técnica tenían que ver con aspectos de la organización, cohesión y participación social de los habitantes; sus habilidades y saberes laborales; su interés y disposición en participar en proyectos de desarrollo social y comunitario; sus percepciones y expectativas del entorno, entre otros.

En la mayoría de los manuales tradicionales de metodologías, se inscribe a las técnicas cualitativas en una fase inicial o exploratoria de una investigación, que pueda aportar elementos para el desarrollo de una investigación cuantitativa, colaborando a ésta en la construcción de posibles hipótesis o preguntas de investigación. En este caso específico, 
Cano-Aguilar

sucede en forma contraria: la recolección de datos cuantitativos que permiten no sólo describir las condiciones del contexto sino también identificar la disponibilidad e interés de las personas, por ejemplo, para participar en acciones en beneficio de la comunidad, antecede a la ejecución e inclusive el diseño, de técnicas cualitativas propias para el nivel comunitario y/o de grupos, como los análisis FODA o los grupos focales.

Se realizó una prueba piloto del instrumento de recolección de información en abril de 2017 con 20 encuestas aplicadas en el centro de la cabecera municipal de Casas Grandes. En este mismo mes se aplicó la encuesta en la colonia Progreso. El área de recolección de información estuvo delimitada por el polígono definido por tres bloques o cuadras hacia cada punto cardinal teniendo como centro el parque de la Colonia (de la Iglesia del Señor de la Misericordia); siendo demarcada esta zona al sur por la calle Paseo del Arte y el área de amortiguamiento natural de la zona arqueológica de Paquimé; al norte hasta la calle Hidalgo; al este, la calle 20 de noviembre; y al oeste, la 18 de marzo. Se recogió información de 86 hogares de 104 habitados en la zona de estudio.

En la recolección de información, el piloteo y la captura de los datos en la base, se contó con la valiosa colaboración del estudiante Josué Quintana y las estudiantes Aylin Ponce, Erika Rodríguez, Paola Carbajal, Daniela Beltrán, Janeth Contreras, Alejandra Martínez, Daniela Montañez, Daniela Amaya, Nahomy Vega, Jacqueline Vega, Guadalupe Madrid, Paola Flores, Aylin Díaz y Ana Carrillo, del curso de Metodología y Práctica de Trabajo Social en Comunidad I (este equipo también participó en la aplicación de las otras técnicas de recolección de datos mencionadas); así como el incondicional apoyo de la Mtra. Gabriela Muñoz García, coordinadora de la carrera de Licenciatura de Trabajo social de la División Multidisciplinaria de la Universidad Autónoma de Ciudad Juárez en Nuevo Casas Grandes, Chihuahua.

Esta técnica aporta elementos para estimar la viabilidad o factibilidad de acciones comunitarias o colectivas, propias de una práctica de Trabajo Social en comunidad. En el nivel de intervención en comunidad, como ya se ha comentado, las acciones, participativas o no, deben atender problemáticas del ámbito comunitario; es decir, la atención de necesidades de salud o educación, tienen como sujeto a la comunidad, no al individuo. En este sentido, cuando se hace el tratamiento de los datos obtenidos a través de una técnica, como la de este caso, se busca información que permite diseñar acciones que impacten en la organización, la participación o el desarrollo comunitario.

\section{Hallazgos}

Se muestran los resultados de la aplicación de este instrumento en tres apartados: características y condiciones de bienestar de los habitantes; su disponibilidad para participar en la organización comunitaria y actividades de promoción social; y sus percepciones y expectativas del "Pueblo Mágico". Esta clasificación se realizó tomando en cuenta los elementos del "espacio territorial que ocupa la comunidad, los habitantes que se encuentran en él, las necesidades que perciben y los recursos con los que cuentan" (Hernández y Braco, 2009, p. 19). Finalmente se señalan áreas potenciales de intervención identificadas.

Prospectiva. Revista de Trabajo Social e intervención social • No. 29 • ene.-jun. $2020 \bullet$ pp. 107-130 e-ISSN: 2389-993X • Doi: 10.25100/prts.v0i29.8196

Universidad del Valle - Cali, Colombia 


\subsection{Características socio demográficas y de bienestar social}

Se recabaron datos de 86 hogares (cerca de 83,0\% del área de estudio), donde viven 297 personas, de las cuales poco más de la mitad son mujeres. La Colonia Progreso es un asentamiento joven, la edad promedio de sus habitantes es de 31 años; una tercera parte tiene 18 años o menos; otra, entre 19 y 40 años; y una tercera parte más entre 41 y 95 años. Las personas mayores de 60 años conforman 7,0\% de la muestra. Con respecto al estado civil de los colonos de la muestra, la mayoría tiene una pareja: están casados $(49,0 \%)$ o en unión libre $(8,0 \%)$; mientras que $35,0 \%$ de las personas dijeron estar solteras, $4,0 \%$ viudas y $2,0 \%$ divorciadas.

En promedio, los habitantes han estudiado 8 años de escuela (sin contar el nivel preescolar), con un nivel de escolaridad de educación básica secundaria incompleta al momento de la encuesta. El porcentaje más alto de escolaridad registrado fue el de primaria completa (seis años: 22,0\%), seguido de secundaria completa (nueve años: 19,5\%) y preparatoria completa (12 años: 8,0\%). Casi la mitad de las personas entrevistadas identificaron alguna necesidad relacionada con la educación en el hogar; de ellas, cerca de $31,0 \%$ dijeron que su principal necesidad era el dinero para pagar cuotas escolares o colegiaturas; $12,0 \%$, que era el dinero para útiles o uniformes; $29,0 \%$, que era el transporte y $12,0 \%$ dijeron que era el tiempo para participar en asuntos de la escuela.

Como se aprecia en la Tabla 1, con respecto a los servicios de salud, poco más de la mitad dijeron contar con un seguro público contributivo (del Instituto Mexicano del Seguro Social, IMSS o del Instituto de Seguridad y Servicios Sociales para los Trabajadores del Estado, ISSSTE); poco más de la tercera parte dijo contar con la asistencia social (Seguro Popular); 6,0\% dijeron no tener ninguno y 2,0\% tener un seguro privado. En cambio, poco menos de la mitad dijeron atenderse en el IMSS o ISSSTE); 35,0\% dijeron atenderse en el Seguro Popular; $11,0 \%$ en un consultorio privado y 3,0\% en una farmacia. Dos de cada tres dijeron que la institución de salud les otorga los medicamentos; y tres de cada diez dijeron obtenerlo con su propio dinero.

Tabla 1. Datos socio demográficos (edad, sexo, estado civil), de escolaridad y servicio de salud de los habitantes

\begin{tabular}{|c|c|c|c|}
\hline $\begin{array}{l}\text { Edad } \\
\text { - } 31 \text { años } \\
\text { Promedio } \\
\text { - } 33,0 \% \text { menores } \\
\text { de } 18 \text { años } \\
\text { - } 66,0 \% \text { menores } \\
\text { de } 40 \text { años } \\
\text { - } 7,0 \% \text {, mayores } \\
\text { de } 60 \text { años }\end{array}$ & $\begin{array}{c}\text { Sexo } \\
\text { - } \quad 53,0 \%, \text { Mujeres } \\
\text { Estado civil } \\
\text { - } \quad 49,0 \% \text { Casados } \\
\text { - } \quad 8,0 \% \text { Unión libre } \\
\text { - } \quad 35,0 \% \text { Solteros }\end{array}$ & \begin{tabular}{l}
\multicolumn{1}{c}{ Escolaridad } \\
- 8 años, Promedio de \\
años de estudio. \\
- $22,0 \%$ con primaria \\
completa ( 6 años) \\
- $19.5,0 \%$ con secundaria \\
completa ( 9 años) \\
- $8,0 \%$ con preparatoria \\
completa ( 12 años)
\end{tabular} & $\begin{array}{l}\text { Servicios de salud } \\
\text { - } 55,0 \% \text { con Servicio } \\
\text { público contributivo } \\
\text { (IMSS - ISSSTE) } \\
\text { - } 37,0 \% \text { con asistencia } \\
\text { pública (Seguro } \\
\text { popular) } \\
\text { - } 2,0 \% \text { con Seguro privado } \\
\text { - } 6,0 \% \text { sin Seguro médico }\end{array}$ \\
\hline
\end{tabular}

Fuente: elaboración propia 
Al preguntarse si alguien en el hogar tiene alguna enfermedad crónica o limitaciones físicas, 46,0\% respondieron que no; 17,0\% dijeron tener hipertensión; 12,0\% diabetes, 8,0\% limitaciones motrices y 4,0\% visuales, auditivas o de lenguaje; 6,0\% dijeron que tienen asma o una enfermedad respiratoria; 3,0\% artritis o reumatismo y 2,0\% dijeron que tenían otro tipo de enfermedad o limitación física. Alrededor del 70,0\% de estas personas con enfermedades o limitaciones dijeron no tener ninguna dificultad o necesidad para atender estos problemas de salud identificados; la principal dificultad reportada fue la del dinero (alrededor del 22,0\%), seguida de no contar con alguien que lo lleve a la consulta médica $(6,0 \%)$.

El promedio de habitantes por hogar es de 3.5, con una moda de $2(28,0 \%)$ y una mediana de 3 habitantes por hogar. En tres de cada cuatro hogares hay 4 habitantes o menos; y en el resto, entre 5 y 9. Cerca de 42,0\% usan dos habitaciones para dormir; $30,0 \%$, una, y 26,0\%, tres. Cerca de 26,0\% de estos hogares tienen tres habitaciones ( $\sin$ contar el baño); $24,0 \%$ cuatro; $21,0 \%$ cinco; $19,0 \%$ dos y 9,0\% seis. En esta colonia, 93,0\% de los hogares tienen el baño en su interior; y 91,0\% cuentan con un patio, solar o jardín. La mayoría son casas con piso de cemento, techos de lámina de metal, y de adobe. Tres cuartas partes de los hogares eliminan sus aguas negras a través de la red pública de alcantarillado, 25,0\% mediante fosa séptica y letrina. El principal combustible para calentar la vivienda es la leña $(74,0 \%)$; y para preparar alimentos, el gas $(91,0 \%)$.

Aproximadamente $80,0 \%$ de las personas que respondieron dijeron que el lugar donde viven es de su propiedad y 5,0\% propiedad de otro miembro del hogar; 5,0\% dijeron que es prestado de un amigo o vecino; 6,0\% prestado de un familiar y sólo 7,0\% rentado. Las personas identificaron como principal necesidad del hogar los alimentos $(51,0 \%)$, los servicios básicos $(10,0 \%)$, las medicinas $(9,0 \%)$ otros servicios $(9,0 \%)$, y las deudas $(7,0 \%)$.

Se pidió a las personas entrevistadas que señalaran en una lista las principales carencias de la colonia. De éstas, como se muestra en la Tabla 2, las calles y el drenaje y agua potable fueron las más señaladas, con $22,5 \%$ cada uno de estos dos rubros. La seguridad pública ocupó el segundo lugar con 17,5\%; el alumbrado 14,0\%; y 11,3\%, las banquetas. Como segunda carencia principal de la colonia, señalaron, en orden de importancia, seguridad pública, banquetas, calles y alumbrado. A una tercera carencia solicitada, fueron señaladas banquetas y calles. Aunque en menor medida, también fueron señalados problemas con la basura y limpieza de la colonia. 
Tabla 2. Tres principales carencias identificadas en la colonia, con base en los porcentajes de las respuestas

\begin{tabular}{lccc}
\hline \multicolumn{1}{c}{ Carencias } & Primera & Segunda & Tercera \\
\hline Calles & $1^{\mathrm{a}}$ & $3^{\mathrm{a}}$ & $2^{\mathrm{a}}$ \\
Agua - drenaje & $1^{\mathrm{a}}$ & & \\
Seguridad pública & $2^{\mathrm{a}}$ & $1^{\mathrm{a}}$ & \\
Banquetas & $4^{\mathrm{a}}$ & $2^{\mathrm{a}}$ & $1^{\mathrm{a}}$ \\
Alumbrado público & $3^{\mathrm{a}}$ & $4^{\mathrm{a}}$ & \\
Limpieza de basura (en “Otras”) & $5^{\mathrm{a}}$ & $5^{\mathrm{a}}$ & $3^{\mathrm{a}}$ \\
\hline
\end{tabular}

\section{Fuente: elaboración propia}

Cuando se les preguntó cómo se vería mejor su colonia o qué le haría falta para mejorar su imagen, 50,0\% respondió que equipamiento (alumbrado, banquetas, señales viales, pavimentación, nombres de calles, etc.), 35,5\%, árboles y 7,0\% las fachadas de las casas. Al pedirles que indicaran otra acción para mejorar la imagen de la colonia, que no estuviera en la lista de respuestas, la mayoría, 75,0\%, indicó que la limpieza. Con respecto a la pregunta sobre si - en el caso de que alguien le ofrezca los materiales y la asesoría estarían dispuestos a hacer mejoras o reparaciones a su hogar con un estilo o diseño definido, por ejemplo, una gama especifica de colores, $79,0 \%$ respondió que sí, 13,0\% no y $8,0 \%$ tal vez.

\subsection{Participación y organización comunitaria}

Una cuarta parte dijo tener 6 años o menos viviendo en esta colonia; 40,0\% dijo tener 10 años o menos; la mitad de ellos dijo tener 15 años o menos viviendo aquí; 75,0\% dijo tener 20 años (ésta fue la edad en la que se encontró la moda) o menos. En promedio, los habitantes tienen 15 años viviendo en esta colonia.

Poco más de dos terceras partes $(68,6 \%)$ de las personas entrevistadas dijeron que en esta colonia viven familiares o paisanos (del mismo lugar de origen). Tal vez debido a esto, casi $85,0 \%$ dijeron participar en fiestas o reuniones familiares; seguido de un 52,0\% dijeron participar en organizaciones religiosas. Asimismo, las reuniones o convivencias más frecuentes $(60,0 \%)$ se dan con familiares o parientes; seguidas de las reuniones con miembros de la iglesia y con amigos.

Es una colonia en la que las relaciones familiares o de parentesco pesan más que otro tipo de relaciones. En la Tabla 3 se presentan los datos alusivos al estado de la organización de la comunidad. Se les preguntó cómo consideran la unión de los vecinos en esta colonia, $56,0 \%$ dijeron que hay poca y $18,0 \%$ que nada, sólo $26,0 \%$ dijeron que era mucha. A la pregunta de si había un comité vecinal en su colonia, 55,0\% dijeron no saber; 39,0\% dijeron que no, y 6,0\% que sí. En realidad, no había, pero más de la mitad de los vecinos desconocían la situación.

Prospectiva. Revista de Trabajo Social e intervención social • No. $29 \bullet$ ene.-jun. 2020 • pp. 107-130 e-ISSN: 2389-993X • Doi: 10.25100/prts.v0i29.8196

Universidad del Valle - Cali, Colombia 
Cano-Aguilar

La mayoría de las personas entrevistadas $(63,0 \%)$ opinaron que con la unión de los vecinos se pueden resolver problemas de seguridad; $19,0 \%$ reconocen problemas de convivencia vecinal y $11,0 \%$ de equipamiento urbano; otros problemas que fueron señalados fueron los de limpieza, control del parque, mejoras en la colonia, quejas contra el municipio, cercos y agua.

Con respecto a la participación, 39,0\% dijeron que nadie en el hogar estaría interesado en formar un comité de vecinos, 26,0\% afirmaron que tal vez, 27,0\% dijeron que sí, y 8,0\% manifestaron que a alguien le interesaría mucho. Los porcentajes de respuestas son similares para la pregunta de si alguien en el hogar se interesaría en integrarse a comités de vecinos y organizaciones existentes $(36,0 \%, 23,0 \%, 29,0 \%$ y $11,0 \%$, respectivamente).

Tabla 3. Información acerca de la organización vecinal en la colonia (porcentajes).

\begin{tabular}{lcccc}
\hline $\begin{array}{l}\text { ¿Hay comité en la } \\
\text { colonia? }\end{array}$ & $\begin{array}{l}\text { Nada } \\
\text { ¿Hay unión } \\
\text { entre los } \\
\text { vecinos? }\end{array}$ & $18,0 \%$ & Poca & Mucha \\
$\begin{array}{l}\text { - No: } 39,0 \% \\
\text { - Sí: } 6,0 \%\end{array}$ & Seguridad & Convivencia & Equipamiento & Otros \\
\hline $\begin{array}{l}\text { Problemas que se } \\
\text { resolverían con la } \\
\text { unión de los } \\
\text { vecinos }\end{array}$ & $63,0 \%$ & $19,0 \%$ & $11,0 \%$ & $7,0 \%$ \\
\hline $\begin{array}{l}\text { Alguien se } \\
\text { interesaría: }\end{array}$ & Nadie & Tal vez & Sí & Mucho \\
$\begin{array}{l}\text { En formar un } \\
\text { comité }\end{array}$ & $39,0 \%$ & $26,0 \%$ & $27,0 \%$ & $8,0 \%$ \\
\hline $\begin{array}{l}\text { En integrarse a un } \\
\text { comité }\end{array}$ & $36,0 \%$ & $23,0 \%$ & $29,0 \%$ & $11,0 \%$ \\
\hline
\end{tabular}

Fuente: elaboración propia

Las personas entrevistadas dijeron que las actividades que realizan con frecuencia son las de estar con amigos, vecinos y familia. Las actividades menos realizadas son las culturales o artísticas, y en las que menos participan los miembros de los hogares visitados. Cuando se les preguntó si alguien del hogar estaría interesado en realizar ciertas actividades de una lista determinada, las actividades donde la mayoría contestó que nadie estaría interesado fueron las de asistir a actividades artísticas o culturales en la colonia. Congruentemente con la falta de interés en actividades artísticas o culturales y la falta de convivencia vecinal o la unión entre vecinos, las personas entrevistadas consideraron que las actividades que menos podrían realizarse en el parque de la colonia son los conciertos o pláticas y las reuniones vecinales. 
Al preguntar si alguien en el hogar está actualmente desempleado y/o si creía que le gustaría seguir trabajando, 80,0\% contestaron que sí. Luego, al preguntar en cuál actividad le gustaría trabajar, las respuestas más altas fueron, como se observa en la Tabla 4: en actividades de taller tradicional (talabartería, mecánica, carpintería, etc.) y de autoempleo (negocio propio).

Alrededor de la mitad dijeron que les gustaría trabajar en actividades agropecuarias, de repostería y cocina, de autoempleo (negocio colectivo), de gobierno, de oficina y de mostrador. Los porcentajes más bajos fueron en oficios artísticos o artesanales y en actividades profesionales e industriales (esta última respuesta coincide con el nivel de escolaridad de la muestra).

Tabla 4. ¿En cuáles actividades le gustaría seguir trabajando?

\begin{tabular}{ll}
\hline Actividades & Porcentaje \\
\hline Agrícolas - pecuarias & $57,4 \%$ \\
De oficina & $49,2 \%$ \\
Profesionales & $44,3 \%$ \\
Gobierno & $54,1 \%$ \\
De taller (Talabartería, mecánica, carpintería) & $72,1 \%$ \\
Oficios (Artísticos o artesanales) & $44,3 \%$ \\
De mostrador & $45,9 \%$ \\
Repostería y cocina & $55,7 \%$ \\
Industriales & $44,3 \%$ \\
Auto empleo (Negocio propio) & $71,7 \%$ \\
Auto empleo (Negocio colectivo) & $54,2 \%$ \\
\hline
\end{tabular}

Fuente: elaboración propia

Al preguntar sobre su experiencia laboral, la mayoría de las personas entrevistadas dijeron tener mucha experiencia en oficios tradicionales (mecánica automotriz, fontanería, electricidad, etc.) y construcción; y nada de experiencia en servicios profesionales y técnicos (por la escolaridad) y en actividades de oficios tradicionales de elaboración de artesanías o bienes rudimentarios; lo que explica parcialmente las respuestas en las preguntas revisadas (ver Tabla 5).

Sumando los porcentajes de las respuestas en los niveles de "poca" y "mucha" experiencia, se advierte que la mayor experiencia de las personas de la muestra se registra en las actividades de "Elaboración y/ o conservas en alimentos regionales o tradicionales", en primer lugar $(77,6 \%)$, después en actividades agropecuarias $(60,0 \%)$, oficios tradicionales como carpintería $(57,6 \%)$, y actividades de la construcción $(55,5 \%)$. 
Tabla 5. Grado de experiencia en actividades relacionadas con...

\begin{tabular}{lccc}
\hline \multicolumn{1}{c}{ Actividades } & \multicolumn{2}{c}{ Grado de experiencia } \\
& Nada & Poca & Mucha \\
\hline $\begin{array}{l}\text { Gastronomía regional (Elaboración y/o } \\
\text { conservas en alimentos regionales o } \\
\text { tradicionales) }\end{array}$ & $22,4 \%$ & $57,6 \%$ & $20,0 \%$ \\
$\begin{array}{l}\text { Oficios (Elaboración de manualidades, } \\
\text { artesanías o bienes rudimentarios) }\end{array}$ & $45,9 \%$ & $31,8 \%$ & $22,4 \%$ \\
$\begin{array}{l}\text { Oficios (Mecánica automotriz, fontanería, } \\
\text { electricidad, etc.) }\end{array}$ & $47,1 \%$ & $25,9 \%$ & $27,1 \%$ \\
$\begin{array}{l}\text { Oficios (Carpintería, repostería, etc.) } \\
\text { Construcción }\end{array}$ & $42,4 \%$ & $34,1 \%$ & $23,5 \%$ \\
$\begin{array}{l}\text { Servicios profesionales } \\
\text { Servicios técnicos }\end{array}$ & $43,5 \%$ & $31,8 \%$ & $24,7 \%$ \\
Agro pecuarias & $76,5 \%$ & $16,5 \%$ & $7,1 \%$ \\
\hline
\end{tabular}

Fuente: elaboración propia

Al preguntar acerca del área de interés para recibir capacitación (Tabla 6), la mayoría de las respuestas se concentraron en la elaboración de alimentos y conservas, oficios tradicionales, reparación y mantenimiento doméstico, y artesanías y manualidades.

Tabla 6. ¿A alguien del hogar le gustaría recibir capacitación en...?

\begin{tabular}{ll}
\hline \multicolumn{1}{c}{ Actividades } & Porcentaje \\
\hline Elaboración de alimentos y conservas & $68,2 \%$ \\
Oficio tradicional & $64,7 \%$ \\
Artesanías y manualidades & $62,4 \%$ \\
Reparación y mantenimiento doméstico & $62,4 \%$ \\
Actividades de construcción & $56,5 \%$ \\
Agricultura- cuidado de animales & $52,9 \%$ \\
Actividades artísticas & $49,4 \%$ \\
\hline
\end{tabular}

Fuente: elaboración propia

Finalmente, como se muestra en la Tabla 7, a pesar de que 9 de cada diez hogares cuenta con patio, solar o jardín, sólo 2 de cada diez entrevistados contestó que en su casa tienen árboles frutales, hortalizas o huerto; y 3 de diez partes dijeron no tener animales de traspatio. Pero el $76,3 \%$ dijo tener interés en tener una siembra de hortalizas o árboles frutales en casa. En tres de cada diez hogares dijeron tener aves de corral, caballos o cabras. 
Tabla 7. Cuenta con huerto e interés en tener uno

\begin{tabular}{cc}
\hline $\begin{array}{c}\text { Tienen en este hogar árboles frutales, } \\
\text { hortalizas o un huerto }\end{array}$ & $\begin{array}{c}\text { Le interesaría tener una siembra de } \\
\text { hortalizas o árboles frutales en su casa }\end{array}$ \\
\hline $21,2 \%$ & $76,3 \%$ \\
\hline
\end{tabular}

Fuente: elaboración propia

\subsection{Representaciones sociales del contexto inmediato}

Cuando se les preguntó cuál era la primera cosa que se le viene a la mente cuando escucha la frase "Casas Grandes", en las respuestas tiene predominancia el Museo y la zona arqueológica de Paquimé - MZA (algunas personas se refieren a esta última como "las ruinas"). En segundo lugar, dominan aspectos subjetivos de la localidad relacionados con la tranquilidad de vivir aquí y la belleza del lugar. Al preguntar por lo segundo que se le viene a la mente al escuchar "Casas Grandes", la tranquilidad y la percepción de belleza ocupan ahora el primer lugar; y el MZA, el segundo. En esta segunda representación, también toma preponderancia el aspecto de la infraestructura física: casas, iglesias, plazas. En ambas representaciones, para los habitantes "Casas Grandes" es representada por la zona turística, la tranquilidad y belleza de la localidad.

Con respecto a las representaciones del término "Pueblo Mágico", volvieron a predominar el MZA; sin embargo, el turismo, las artesanías y la proyección hacia afuera de la localidad sobresalen en segundo lugar. En la segunda representación de "Pueblo mágico", sobresalen también el turismo, las fiestas y las artesanías; luego, la posibilidad de más atención por parte del gobierno y mejoras en la imagen, así como la belleza del lugar.

Al preguntar lo que consideraban como distintivo de Casas Grandes, la primera respuesta estuvo dominada por el MZA, reafirmando la preponderancia de este lugar y su cercanía física a la colonia. En segundo lugar, se encuentran las respuestas asociadas al turismo, las artesanías, las fiestas y tradiciones. No señalaron aspectos negativos. También, al preguntar por la segunda cosa que consideran distintiva de Casas Grandes, sobresale el MZA, y en segundo lugar las artesanías y aspectos relacionados al turismo, aunque también se señalan las personas y su bonhomía.

En la exploración de representaciones y percepciones ligadas al turismo, de nueva cuenta indicaron que un primer sitio para visitar con la familia en Casas Grandes o la región es el MZA; seguido de otra zona arqueológica (la Cueva de la Olla); y finalmente, las plazas y parques y los sitios de recreación acuáticos (río, albercas, el balneario Ojo Vareleño u Ojo de San Antonio), tal vez por haberse recogido la información en la mitad de la primavera (abril). Un segundo sitio para visitar con la familia en la localidad, también fue el MZA y la Cueva de la Olla; sin embargo, en esta segunda respuesta los lugares con agua son los más mencionados. También sobresalen los parques y algunas localidades del municipio (Colonia Juárez, Mata Ortiz y Colonia Pacheco). 
Con respecto a los sitios que consideran que deben visitar los turistas foráneos, de nueva cuenta sobresalen los sitios arqueológicos; y los sitios históricos como Mata Ortiz y las Ex Haciendas. Al final señalan los balnearios. También los sitios arqueológicos sobresalen en la segunda respuesta (encabezados por la Cueva de la Olla), seguidos de las localidades cercanas (Colonia Juárez y Mata Ortiz); ahora los balnearios también sobresalen, seguidos de los espacios públicos como plazas y parques. Los lugares menos conocidos por los miembros de las familias de la muestra en esta colonia, fueron la Casa del Nopal (en el centro de la cabecera); la Casa de las botellas o Recinto del Arte (en la misma colonia); y la Ex Hacienda de San Diego (fuera de la cabecera).

Las personas entrevistadas consideran que las principales ventajas que pueden traer a la colonia el desarrollo del turismo, están relacionadas con la economía: progreso, dinero, empleo, negocios, ingresos, etc. En la primera respuesta también resaltan ciertos beneficios no económicos, relacionados con la infraestructura local y ayudas o beneficios para la comunidad.

Finalmente, se encontró que los vecinos de esta colonia ven como una manera de aprovechar el desarrollo del turismo la realización de actividades principalmente de servicios de hospedaje, alimentos y comercio y, en segundo lugar, beneficios económicos vinculados al empleo y el crecimiento económico. Otras actividades, ligadas también al turismo, fueron señaladas: producción y venta de artesanías y guía de turistas. Cabe señalar que un $10,0 \%$ de la muestra indicaron que no sentían que el turismo pudiera beneficiarles en algo.

\subsection{Potenciales áreas de intervención identificadas}

Con base en los resultados expuestos, a continuación, se presentan agrupaciones de posibles áreas identificadas para incidir en la situación de organización y cohesión comunitaria, las condiciones vigentes y posibilidades de lograr la participación de los colonos en el desarrollo comunitario, y la viabilidad de realizar acciones colaborativas asociativas con este mismo fina.

1. Una primera dimensión es la del bienestar social, equipamiento y acción social. Con respecto a las necesidades de bienestar y áreas de intervención derivadas de las características de la población, se encontró que esta dimensión no presenta problemas de urgente atención: las condiciones y necesidades de educación, salud y vivienda están cubiertas en niveles mayores a los satisfactorios. Por otro lado, se identificaron un conjunto de necesidades y carencias que tienen que ver con el entorno físico de la comunidad, que implica la acción pública y social. Los servicios básicos, particularmente calles, banquetas, alumbrado y drenaje, y agua potable fueron identificados como las principales carencias de la colonia, seguidos de la seguridad pública. Se hizo también hincapié en mejorar la calidad de los servicios municipales de recolección de basura y limpieza de la colonia. Para mejorar la imagen de la colonia, la mitad de los entrevistados señaló la 
atención a estas carencias de equipamiento urbano y en la limpieza, y una tercera parte, plantar árboles.

Aquí hay un área de oportunidad muy concreta y específica para los diferentes niveles de gobierno, localizada en la mejora e introducción de equipamiento urbano con un estilo ad hoc al museo y zona arqueológica, dado que este rubro fue identificado como una carencia de la colonia, por un lado, y por estar ésta ubicada frente al MZA y contar con el parque y la iglesia del Señor de la Misericordia y el Recinto del Arte, atractivos arquitectónicos para visita de personas de la región y foráneos.

En el marco de un proyecto de desarrollo comunitario, esta sería una acción de mediano plazo y subsecuente al logro de un nivel mínimo de organización y participación de los miembros de la comunidad, con una mayor participación de agentes externos, como las instancias públicas de la municipalidad o las organizaciones civiles o no gubernamentales. Sin embargo, como se mencionó al inicio de este trabajo, el proyecto en el que se enmarcó esta técnica es más acorde a un modelo de desarrollo desde adentro y con la participación de los integrantes de la comunidad.

2. En este sentido, un segundo campo de aportaciones al diagnóstico lo conforma la organización y participación comunitarias, del cual se advierten claramente áreas específicas de intervención. Se puede ver como una oportunidad para la acción, la percepción extendida entre los entrevistados de que hay poca o nula unión entre los vecinos; y se debe considerar la percepción positiva de la mayoría de los entrevistados acerca de que esta unión entre vecinos resolvería problemas de inseguridad, convivencia vecinal y falta de equipamiento urbano. Hay una carencia identificada y también la percepción del beneficio o relevancia que significa cubrirla.

También se deben considerar dos datos importantes: el interés y disposición de los vecinos y la proporción entre convivencia familiar y vecinal. Cuatro de cada diez dijo que nadie en el hogar estaría interesado en formar o participar en un comité vecinal, lo que abre un campo para la intervención social en actividades de información, sensibilización y promoción social. Por otro lado, se abre también una oportunidad para acciones que propiciarían un incremento en la frecuencia de convivencia entre vecinos, pues los hábitos de convivencia actuales están dominados principalmente por el ámbito familiar; esto implicaría actividades de convivencia, artísticas y culturales en ciertos espacios de la colonia. 
Cano-Aguilar

En este sentido, de acuerdo a los datos de la muestra, es factible implementar una campaña de reforestación de especies nativas en esta colonia, mediante un apropiado y pertinente proyecto de participación comunitaria. También hay posibilidades de plantear un proyecto comunitario de huertos domésticos, justificado porque, de acuerdo con los datos expuestos, la mayor necesidad en el hogar fue la alimentación; más de la mitad dijeron estar interesados en trabajar en actividades agropecuarias o poner un negocio propio o colectivo; y nueve de cada diez hogares cuentan con patio, solar o jardín. La propuesta es además viable porque en ocho de cada diez hogares visitados dijeron no tener árboles frutales, hortalizas o huerto; y alrededor de dos terceras partes dijeron no tener animales de traspatio; y sin embargo en casi ocho de cada diez dijeron tener interés en tener hortalizas o huerto.

3. Es posible promover también la capacitación en oficios artesanales y artísticos, debido a la ubicación de la colonia, la escolaridad de los habitantes y sus expectativas. Al preguntar acerca del área de interés para recibir capacitación, la mayoría de las respuestas se concentraron en la elaboración de alimentos y conservas, oficios tradicionales, reparación y mantenimiento doméstico y artesanías y manualidades. Estas actividades, además de las agropecuarias y de construcción, obtuvieron los mayores porcentajes en el nivel de "poca" experiencia de la pregunta anterior. Esto quiere decir que tienen poca experiencia en estas actividades, pero que hay interés en capacitarse en ellas. Por la experiencia laboral, hay una inclinación a capacitarse y desarrollarse laboralmente en un negocio propio como mecánico, fontanería, electricidad, etc. Sin embargo, hay un área de oportunidad en la alta disponibilidad e interés que mostró la mayoría de los entrevistados, en recibir capacitación en actividades como elaboración de alimentos tradicionales y conservas, oficios, reparación y mantenimiento doméstico, y artesanías y manualidades; esta alta disponibilidad identificada, puede tener alguna asociación relevante con las percepciones y representaciones de los habitantes, presentadas en el apartado 3.3 de este artículo, asociación que deberá ser explorada a través de las técnicas cualitativas subsecuentes.

4. Para los habitantes entrevistados, la vecina zona arqueológica con su museo de sitio, visitado por turistas locales, nacionales y extranjeros, es el principal referente en las frases propuestas: "Casas Grandes" (además de la tranquilidad y belleza de la localidad); "Pueblo Mágico" (junto con actividades de turismo foráneo y artesanías); fue considerado el primer sitio para visitar con la familia. Aunadas estas percepciones al tiempo promedio que tienen viviendo en la colonia (arraigo), hay un recurso latente, que contribuiría, entre otras cosas, a 
crear y fortalecer una identidad comunitaria por su ubicación física, aledaña a Paquimé.

Con respecto al desarrollo del turismo, las personas entrevistadas percibieron como sus principales ventajas el progreso, dinero, empleo, negocios, ingresos, etc., y se registró una considerable disposición a aprovechar las actividades del sector: hospedaje, alimentos, artesanías, etc. Las acciones que se implementen en estas potenciales áreas de intervención estimadas, ayudarían a convertir en una oportunidad, adecuada al contexto, la falta de interés y frecuencia de actividades artísticas y culturales, precisamente en el espacio que consideran menos adecuado para ello: el parque de la plaza del Señor de la Misericordia, en el centro de la colonia.

De este modo, con la información obtenida de la técnica de encuesta, se pudieron identificar áreas de oportunidad para incidir en los niveles de organización y participación comunitaria (numeral 2), la promoción social (numeral 3) y la cohesión e identidad comunitarias (numeral 4), aspectos inherentes al desarrollo comunitario; y de este modo, trascender el papel del Trabajo Social de comunidad como mera gestión de recursos externos o acción social para el equipamiento urbano (numeral 1).

\section{Conclusiones}

Antes de desarrollar este último apartado, se deben considerar dos advertencias importantes. Primero, hay que tomar en cuenta que no se habían hecho diagnósticos de este tipo (ni intervenciones sociales) en esta colonia. Y segundo: si bien se exponen elementos obtenidos exclusivamente a partir de la encuesta, aún quedaría por examinar y valorar los datos que aportarían otras técnicas y fases del proyecto general de diagnóstico, referidos en el apartado metodológico, pues como se ha dicho ya, esta técnica conforma sólo una de las fases de la construcción del diagnóstico integral. Además, haría falta aplicar otras técnicas, cualitativas, para profundizar en las áreas identificadas de problemas y construir los diagnósticos para la intervención.

Para completar este diagnóstico, se requieren, por ejemplo, entrevistas semiestructuradas, grupos nominales, análisis FODA y entrevistas en profundidad. Lo presentado en este trabajo es sólo un paso en un proceso más amplio, en el que los datos cuantitativos ayudan a identificar en las comunidades carencias y problemas, pero también recursos y subterfugios; el siguiente paso consistirá en realizar un diagnóstico participativo con apoyo en técnicas cualitativas, que permita completar el proceso y conducir a un diagnóstico integral que sirva de fundamento para proyectos factibles de intervención para el desarrollo comunitario. Por lo tanto, lo que a continuación se expone debe tomarse como aportaciones al diagnóstico y no cómo el diagnóstico mismo.

Prospectiva. Revista de Trabajo Social e intervención social • No. $29 \bullet$ ene.jun. $2020 \bullet$ pp. 107-130 e-ISSN: 2389-993X • Doi: 10.25100/prts.v0i29.8196

Universidad del Valle - Cali, Colombia 
En el ámbito del bienestar social, son las carencias de urbanización las que requieren de un proyecto de acción social para la comunidad, en el que el Trabajador Social juega roles de gestor, mediador e implementador, entre la comunidad y el gobierno o instituciones civiles; realizando acciones para gestionar servicios públicos, equipamiento urbano, para acercar servicios generales de salud, etc. Este papel depende del Trabajador social y sus habilidades, y la participación de los habitantes es prácticamente insignificante (aunque esta participación facilitaría la acción social).

En cambio, en el plano del desarrollo comunitario, para poder impulsar cualquier proyecto es necesario trabajar primero en el nivel de la organización comunitaria, y ésta no existe en la colonia El Progreso. Con la organización comunitaria, el papel del Trabajador social trascendería de la acción social a la de promoción y el desarrollo comunitario. Para completar el diagnóstico en este nivel, tendría que entrevistarse a las personas que dijeron estar más interesadas y realizar técnicas grupales participativas para la definición de problemas y el reconocimiento de recursos en la colonia.

La organización y participación de los colonos, podría ser un recurso efectivo para la realización y el mantenimiento de acciones vinculadas a la limpieza, seguridad y obras de equipamiento de la colonia. Estas áreas de intervención, desde el nivel de la participación comunitaria, tienen relevancia pues atenderlas equipararía a la colonia con la representación de Casas Grandes como lugar bello y tranquilo.

Aunado a esto, hay buena disposición de los habitantes, para emprender acciones relacionadas con la unidad doméstica, ya sea para mejorar su aspecto físico o para el aprovechamiento de los patios o solares con los que cuentan la mayoría de las viviendas, en la creación de huertos familiares. Asimismo, para acciones de promoción social que impacten en desarrollo comunitario, se cuenta con la disposición e interés de los habitantes para capacitarse o emprender algún oficio o negocio, y sus trayectorias laborales.

Finalmente, se pueden identificar las aportaciones y potencialidades de esta técnica, en la forma en que se ha presentado en este artículo:

a. otorga una descripción general y panorámica de los habitantes de la comunidad, de sus condiciones de vida y su estructura sociodemográfica, aspectos estructurales que deben considerarse en el diagnóstico comunitario formal.

b. metodológicamente, posibilita a los y las practicantes, recorrer palmo a palmo el área de estudio e intervención, familiarizarse con los puntos de referencia espaciales y temporales (horarios), hacerse visibles y adoptar un papel en el espacio, identificar porteros y personajes clave, conocer las principales dinámicas, entre otros.

c. estimar la situación de elementos fundamentales para el desarrollo comunitario, como son la cohesión social, la intensidad y contenido de las relaciones entre los vecinos, la frecuencia e intencionalidad de las dinámicas 
Cano-Aguilar

de convivencia en la comunidad, el tipo y alcances de la identidad comunitaria, etc.

d. identificar puntos de partida para la acción y dificultades y obstáculos que afectan la viabilidad de la intervención social.

e. contar con información acerca de la disponibilidad, disposición e interés de los integrantes de la comunidad, para la realización de acciones asociativas para la promoción social, fortalecimiento de la identidad y participación comunitaria.

f. identificar a personas con alta disponibilidad e interés, que propicien catalizar procesos de organización, participación y desarrollo comunitarios.

g. conformar una base heurística para el desarrollo de las subsecuentes e imprescindibles técnicas cualitativas requeridas para la construcción y terminación del diagnóstico social integral de la comunidad.

Una vez completado el diagnóstico integral, se puede diseñar y echar a andar un proyecto, en el que los miembros de la comunidad, al mismo tiempo que se van integrando a las actividades propuestas, se van apropiando de ellas, incrementándose su participación al grado que la comunidad, de ser una mera vecindad de colonos, se convierte en el motor del cambio y desarrollo.

\section{Referencias bibliográficas}

Aguilar, M., y Ander-Egg, E. (1999). Diagnóstico social. Conceptos y metodología. Argentina: Hvmanitas.

Arteaga, C., y González, M. V. (2001). Diagnóstico. En C. Arteaga, Desarrollo comunitario (pp. 82-106). México: UNAM. Recuperado de https://trabajosocialmazatlan.com/multimedia/files/InvestigacionPosgrado/D iagnostico \% 20Carlos\%20Arteaga.pdf.

Barbero, J., y Cortés, F. (2005). Trabajo Comunitario, organización y desarrollo social. España: Alianza Editorial.

Cano, A., Estrada, M., y Chávez, G. (2017). Violencia y adolescentes en el noroeste de Chihuahua. Percepciones y experiencias de escolares de secundaria en el período 2007-2012. En P.E. Angarita y J. Vega (Eds.), Violencia, seguridad y derechos humanos (pp. 167-190). Colombia: Universidad de Antioquia. Universidad CES. Universidad de Guanajuato.

Carballeda, A. (2013). La Intervención Comunitaria: una mirada a algunos aspectos contextuales y metodológicos. Revista Abordajes, 1(1), 4-14.

Colorado, A. (2015). Planeación y gestión municipal. En SECTUR, Talleres de Inducción a Pueblos Mágicos y de Planificación y Gestión para el Desarrollo Turístico Municipal. México: Secretaría de Turismo. Programa Pueblos Mágicos.

Crespo, M. (2011). Guía de diseño de proyectos sociales comunitarios bajo el enfoque del marco lógico: (Compendio de conceptos esenciales y aplicaciones). Recuperado de http:/ / www.eumed.net/libros-gratis/2015/1487/index.htm\#indice.

Prospectiva. Revista de Trabajo Social e intervención social • No. $29 \bullet$ ene.-jun. 2020 • pp. 107-130 e-ISSN: 2389-993X • Doi: 10.25100/prts.v0i29.8196 Universidad del Valle - Cali, Colombia 
Cury, S., y Arias, A. (2016). Hacia una definición actual del concepto de "diagnóstico social". Breve revisión bibliográfica de su evolución. Alternativas. Cuadernos de Trabajo Social, (23), 9-24.

Eito, A., y Gómez, J. (2013). El concepto de comunidad y el Trabajo Social. Espacios Transnacionales, (1), 10-16. Recuperado de http:/ / espaciostransnacionales.org/wp-content/uploads/2014/10/1Conceptotrabajosocial1.pdf.

Hernández, J., y Braco, L. (2009). El Trabajo Social Comunitario en España: una aproximación a la historia y a la actualidad. En J. Hernández (Comp.), Trabajo social comunitario en la sociedad individualizada (pp. 17-38). España: Nau Llibres.

INAH. (2016). Estadísticas de visitantes en el estado de Chihuahua, junio y diciembre de 2015. Recuperado de http:/ / www.estadisticas.inah.gob.mx/.

INEGI. (2010). Censo Nacional de Población y Vivienda 2010. México: Instituto Nacional de Estadística, Geografía e Informática.

Instituto de Investigación y Desarrollo Tecnológico para la Agricultura Familiar - IPAF NOA INTA. (2010). Guía para formulación participativa de proyectos en organizaciones de base comunitaria. Argentina: Instituto de Investigación y Desarrollo Tecnológico para la Agricultura Familiar (IPAF NOA INTA).

Lillo, N., y Roselló, E. (2004). Manual para el Trabajo Social Comunitario. España: Narcea.

Maldonado, A. (2013). Sistematización de la práctica de Metodología de Comunidad, realizada en la colonia Emiliano Zapata, en Nuevo Casas Grandes, Chihuahua, en el semestre de enero - junio de 2012 (Tesis de pregrado). Universidad Autónoma de Ciudad Juárez, Juárez, México.

Maya, I. (2016). 7 usos del análisis de redes en la intervención Comunitaria. REDES. Revista Hispana para el Análisis de Redes Sociales, 27(2), 1-10.

Millán, L., y Pinedo, K. (2014). Diagnóstico comunitario e intervención social en la comunidad La Victoria. Sistematización de una experiencia. Interacción y Perspectiva. Revista de Trabajo Social, 4(1), 84-99.

Mori, M. (2008). Una propuesta metodológica para la intervención comunitaria. Liberatbit, 14(14), 81-90.

Ruiz, B. L. (2016). Intervención social de la colonia La Esperanza, en Casas Grandes, Chihuahua. Movilizando a la comunidad para el equipamiento urbano (Tesis de pregrado). Universidad Autónoma de Ciudad Juárez, Juárez, México.

SECTUR. (2006). Reglas de Operación Pueblos Mágicos. México: Secretaría de Turismo.

SECTUR. (2015). Guía de incorporación y permanencia Pueblos Mágicos. México: Secretaría de Turismo.

SECTUR. (2017). Guía para la Integración documental Pueblos Mágicos 2017. México: Secretaría de Turismo.

Viscarret, J. J. (2009). Modelos de intervención en el Trabajo Social. En T. Fernández (Coord.), Fundamentos del Trabajo Social (pp. 345-384). España: Alianza 


\section{OTROS ARTÍCULOS DE PROSPECTIVA No. 29 DE 2020}

EDITORIAL

La Reconceptualización: una opción a la encrucijada del Trabajo Social en Colombia Víctor Mario Estrada-Ospina

\section{ARTÍCULOS}

Formación profesional y la práctica supervisada en el Servicio Social brasileño

Reginaldo Ghiraldelli

El rol docente del tutor y supervisor de prácticas de Trabajo Social: construcción de la reflexividad y el compromiso durante la formación a través de la supervisión

Carmina Puig-Cruells

Problematizando las prácticas preprofesionales en Trabajo Social. Desafios y perspectivas

Ruth Noemí Parola

Trabajo Social en contextos de formación política: la práctica transformadora

Angélica López-Yepes

Jackeline Cuenca-Echeverry

Investigación cuantitativa en una práctica universitaria de intervención en comunidad en el norte de México

Adán Cano-Aguilar
Interventores y comunidades vulnerables: una propuesta de formación para universitarios

Dulce Nayeli Cohetero-Hernández

Fidel González-Quiñones

Reagrupación familiar de menores en Aragón. Propuestas de acompañamiento en el proceso migratorio

Chabier Gimeno-Monterde

José David Gutiérrez-Sánchez

Aportaciones de la Educación en Derechos Humanos (EDH) y la Investigación Acción Participativa (IAP) en contextos de trabajo comunitario

Carla Cubillos-Vega

Condiciones para una NeoReconceptualización del Trabajo Social en Chile, Latinoamérica y el Caribe

Luis Vivero-Arriagada

Justicia sin daño: una apuesta por el fortalecimiento del acceso a la justicia Diego Fernando Acosta-Daza

\section{IN MEMORIAM}

Camilo Bautista Ochoa, un santandereano que se enamoró del Pacífico

Jesús Glay Mejía-Naranjo

\section{PROSPECTIVA}

Revista de Trabajo Social e Intervención Social 